\title{
Anti-FL(FITC-E2) CAR T Cells
}

National Cancer Institute

\section{Source}

National Cancer Institute. Anti-FL(FIT C-E2) CAR T Cells. NCI Thesaurus. Code C158099.

A preparation of genetically modified T-cells transduced with a replication incompetent, self-inactivating (SIN) lentiviral vector expressing a second generation chimeric antigen receptor (CAR) consisting of an anti-fluorescein (anti-FL) fluorescein isothiocyanate (FITC)-E2 single chain variable fragment (scFv), that is coupled, via an immunog lobulin G4 (IgG4) hinge-CH2(L295D)-CH3 spacer, to the costimulatory signaling molecules CD28, CD137 (4-1BB), and CD3 zeta, and linked to a truncated form of the human epidermal growth factor receptor (EGFRt), with potential immunostimulating and antineoplastic activities. Prior to the administration of anti-FL(FIT C-E2) CAR T-cells, the CAR-T adaptor molecule (CAM) EC17 is administered. EC17 is a bispecific molecule that is composed of folic acid conjug ated to FITC (folate-FITC). EC17 targets and binds with its folate moiety with high affinity to folate receptor (FR)-expressing tumor cells. Upon administration of the anti-FL(FITC-E2) CAR T-cells, these cells are attracted by and bind to the FITC antigen moiety of EC17. Upon binding to EC17, the T-cells induce specific tumor cell lysis, cytokine secretion, and proliferation, and activate a robust immune response against the EC17-bound, FR-expressing tumor cells. FR is overexpressed in various tumor cell types and is associated with increased leukemic cell proliferation and aggressiveness. The costimulatory molecules are required for full T-cell activation and enhance both proliferation of T-cells and antitumor activity. EGFRt both facilitates detection of the administered T-cells in vivo and can promote elimination of those cells following a cetuximab-induced antibody-dependent cellular cytotoxicity (ADCC) response. The reactivity of the anti-FL(FITC-E2) CAR T-cells is dependent on dosing of EC17, and therefore allows CAR T-cell activity to be controlled by dosing of EC17. 